REVISTA ANDALUZA DE ANTROPOLOGÍA.

NÚMERO 3: MIGRACIONES EN LA GLOBALIZACIÓN.

SEPTIEMBRE DE 2012

ISSN 2174-6796

[pp. 208-227]

http://dx.doi.org/10.12795/RAA.2012.i03.10

\title{
ACULTURACIÓN Y DIFERENCIACIÓN SOCIAL EN TANGANYIKA COLONIAL: EL IMPACTO DE MEDIDAS DE DESARROLLO SOCIALES EN LAS IDENTIDADES DE GÉNERO AFRICANAS
}

ROSER MANZANERA RUIZ

Grupo de Investigación AfricaInes. Universidad de Granada

\section{Resumen.}

Las medidas de desarrollo coloniales británicas dirigidas a las mujeres impactaron de manera desigual en ellas, influyendo en las relaciones de género. Estas medidas han supuesto procesos de aculturación en varios sentidos: por una parte, por la transformación del modelo femenino y familiar africano "tradicional"; por otra parte, por el fomento de la diferenciación social entre las mujeres que las situará en posiciones diferentes en el movimiento independentista en Tanganyika y, en la posterior etapa postcolonial. El presente artículo rescata datos etnohistóricos de la investigación de campo desarrollada en Tanzania, durante los años 2006 y 2007, para mostrar la influencia de las ideologías europeas sobre la posición social de las mujeres y en la configuración de las nuevas clases sociales que conformarán el país tras su independencia.

Palabras clave: Identidad de género, procesos de aculturación, políticas coloniales, resistencia de mujeres, Tanzania, África. 


\begin{abstract}
.
British colonial development policies targeted at women impacted them in an unequal ways and, influencing gender relations. These measures have resulted in different forms of acculturation's processes: in the one hand, they influenced the "traditional" African feminine and familiar model transformation; in the other hand they promoted social differentiation among women, situating them in different positions in the independents movement in Tanganyika and, after independence. This document recovers ethnographic data from fieldwork in Tanzania, during 2006 and 2007, showing that European gender ideologies influenced the status of women and the new social class that shape the country after independence.
\end{abstract}

Keywords: Gender identity, acculturation process, colonial policies, women's resistances, Tanzania, Africa.

\title{
1. INTRODUCCIÓN
}

Las identidades de género africanas se alteraron en el contacto con nuevas concepciones de feminidad introducidas desde medidas de desarrollo colonial. En el continente africano, la categoría género adquirió significado por dos hechos fundamentales: el primero, en contacto con las ideologías de género hegemónicas que los colonos introdujeron y, el segundo, desde los discursos feministas europeos en la construcción de la categoría "mujer" (Oyewumi, 1997; Nzegwu, 2000; Vieitez, 2005). La categoría género en Europa ha sido entendida como la oposición entre lo masculino y lo femenino (Rosaldo, 1974; Moore, 1991), y tal oposición no existía en periodos pre-coloniales en África (Oyewumi, 1997). El género como categoría social de análisis es en un instrumento fundamental para desvelar las relaciones de poder introducidas a partir de estas incursiones coloniales en el continente africano que alteraron de manera negativa el estatus social del que gozaban las mujeres en periodos anteriores. Ejemplos de ello son los cambios que se introdujeron en la división sexual del trabajo en las tareas agrícolas y, en el acceso y control diferencial sobre los recursos económicos y sociales (Boserup, 1970; Berger y White, 1999).

Las medidas de desarrollo colonial británico significaron procesos de aculturación entendidos como aquellos por los cuales, a través del tiempo, un grupo cultural adopta las creencias y prácticas de otra cultura y, de distinción social, a través de los cuales se fomentó la estratificación social, religiosa, de clases y de género. En este artículo nos centraremos en el análisis de medidas coloniales dirigidas a la promoción de las mujeres tanzanas a partir de la segunda mitad del siglo XX, a través de un estudio de caso etnohistórico en Lushoto, al norte del país: la educación femenina y los clubes de mujeres. 
Las medidas sociales conocidas como desarrollo rural social en Tanganyika tuvieron su origen en 1949, a través de la implantación de centros de bienestar y del Programa de Alfabetización en las montañas Pare, en la zona norte. Posteriormente, se incorporaron como parte del programa los clubes femeninos, extendiéndose ambas medidas al resto del territorio. Sus objetivos eran mejorar los estándares de vida de la población rural mostrando "la importancia de la higiene en la casa, de la nutrición, de la conservación de la tierra y el agua, y el uso y valor de la alfabetización y conocimiento de los asuntos actuales" (Informe anual de Desarrollo Social 1953-1954).

Los clubes de mujeres se fundamentaron en las ideologías de género europeas de la época. En estos clubes se enseñaba fundamentalmente cómo cuidar de los hijos y del hogar. La promoción de las mujeres y la elevación de su estatus, según estas ideologías fomentaban la reclusión femenina en espacios exclusivamente domésticos y privados, donde sus principales responsabilidades eran los trabajos domésticos. Ambas medidas buscaban en definitiva el establecimiento de un modelo femenino para la provisión de esposas a la nueva élite de hombres africanos según los "estándares" de ese momento (Rogers, 1980; Berger y White, 1999).

En este artículo se analizan estas medidas de desarrollo social para mostrar primero, cómo fomentaron determinados procesos de aculturación que implicaron una importante diferenciación social, religiosa, de clase y de género y, segundo cómo influyeron en el distinto papel que jugaron las mujeres cristianas e islámicas en el movimiento independentista y, en el estatus social femenino en periodos posteriores. Para alcanzar estos objetivos este artículo se divide en cuatro partes, a saber: en la primera parte, se analiza la influencia de estas medidas en la definición de un nuevo estatus social para las mujeres africanas. En la segunda parte, se identifica cómo estas medidas introdujeron una nueva forma de entender el papel de las mujeres en la sociedad que favoreció por un lado, las ideologías patriarcales existentes, y por otro, a aquellas mujeres cristianizadas, generalmente hijas o esposas de líderes tanzanos y funcionarios al servicio colonial, frente a las mujeres islámicas de las zonas rurales. En la tercera parte, se expone cómo estas mujeres islámicas que quedaron excluidas, formaron parte del movimiento independentista en Tanganyika haciendo frente al doble sistema patriarcal impuesto en este periodo: el existente en su propia sociedad y el nuevo originario de Europa.

\subsection{Metodología}

Para llevar a cabo esta investigación se utiliza, principalmente, metodología cualitativa, a través de trabajo de campo etnográfico y trabajo de documentación y archivo. A fin de obtener información sobre las medidas de desarrollo social y su influencia en la vida de las mujeres, sobre los intereses y roles femeninos, se realizaron entrevistas de vida a ocho mujeres participantes y no participantes en los clubes, en edades de participar en las medidas de desarrollo social, cuatro de ellas cristianas y cuatro islámicas. Se realizaron 
doce entrevistas abiertas a hombres y mujeres para buscar evidencias sobre los efectos de las medidas de desarrollo coloniales en la división sexual del trabajo en los hogares y comunidades.

El trabajo documental se realizó en las oficinas de desarrollo agrícola y de desarrollo comunitario del distrito de Lushoto, en el Archivo Nacional del país situado en la capital Dar es Salaam y en los centros documentales de la Universidad de Dar es Salaam.

\section{EL ESTATUS DE LAS MUJERES EN LAS MEDIDAS DE DESARROLLO SOCIAL}

El estatus entendido como indica su raíz latina como el estado de algo dentro de un marco de referencia dado, se ha referido a las mujeres como la posición social de éstas dentro de un grupo de personas, familias, o comunidades. Cuando es referido a la posición social de las mujeres en sus comunidades se habla de un estatus adscrito por razón de género. En este sentido podemos definir el estatus como un fenómeno que emerge desde la organización social, a través de un proceso de diferenciación y evaluación, más que el crecimiento de una característica intrínseca o natural de un ser humano per se (Zelditch, 1968). La institucionalización de tales procesos de diferenciación y valoración basadas en las diferencias sexuales construyen un elaborado sistema de diferenciación de género (Moore, 1991) y configuran una localización diferente del estatus para hombres y mujeres (Bradely y Khor, 1993). En ciencias sociales, el estatus de las mujeres ofrece dos significados conceptuales. Por una parte, en el sentido de una agrupación de derechos $y$ deberes asociados a unas posiciones particulares (Linton, 1936). Por otra parte, el concepto se refiere al lugar de las mujeres en relación con los hombres en una jerarquía de dos, implicando estratificación. En este sentido, la aplicación de esta noción de estratificación sexual para describir las relaciones entre hombres y mujeres en las sociedades africanas ha sido cuestionado por diversos estudios que muestran que una posición favorable paras las mujeres en cualquier dimensión de la vida no implica necesariamente una situación igual en otra dimensión (Whyte, 1978; Sudarkasa, 1986; Iniesta, 2010).

En el contexto africano, se ha asumido generalmente, que el problema de las mujeres en sus sociedades proviene de su estatus, entendiéndolo con un único significado, lo que ha supuesto la intervención de planificadores del desarrollo occidentales con el fin de elevarlo a partir de esta idea de estratificación que entiende que sus posiciones sociales, económicas y políticas estaban en una escala inferior a la de los hombres (Rogers, 1980).

Tal definición ha tenido varias consecuencias: Primero, que las mujeres africanas hayan sido percibidas por el resto del mundo como una única categoría, homogénea, sin distinciones de clase, grupo étnico, edad, religión... Segundo, que las mujeres 
africanas hayan sido representadas cómo mujeres sin capacidad de empoderamiento ${ }^{1}$, entendiéndolo cómo el proceso por el cual aquellos a los que se les ha negado el poder obtienen poder, en concreto, las habilidades particulares de realizar elecciones estratégicas sobre sus vidas (Kabeer, 2001; Cornwall y Brock, 2005). Por tanto se las ha conceptualizado cómo victimas pasivas de las situaciones de inferioridad dado su estatus adscrito y, consecuentemente, sujetos receptores de ayuda. Tercero, esta concepción sobre el estatus de las mujeres ha impactado enormemente en las políticas sociales y económicas diseñadas por una parte, para la lucha contra la pobreza, y por otra, para el empoderamiento femenino, excluyéndolas de los beneficios de la economía comercial y del empleo formal.

La ideología sobre el destino doméstico de las mujeres predominó en las primeras medidas de desarrollo social, a través de la enseñanza de saberes que estos clubes implantaron, basadas en el tipo de habilidades domésticas europeas, y de una moralidad queles enseñaba que el lugar adecuado de las mujeres estaba en sus hogares y comunidades. Los clubes mantenían la idea de que las mujeres africanas estaban en posiciones de inferioridad con respecto a los hombres. Desde las ideologías de género de estos clubes se esperaba que cambiando determinados roles femeninos cambiaría automáticamente su estatus, liberándolas de las tareas agrícolas, de la ignorancia... en la que se encontraban (Berguer y White, 1999). El problema principal de esta concepción sobre el estatus femenino proviene primero, de la falta de conocimiento sobre las diversas situaciones en que las mujeres se encontraban. Presuponer que el cambio de algunos de los comportamientos esperados de las mujeres tanzanas cambiaría sus estatus oculta innumerables factores relacionados con la posición de éstas no sólo en sus sociedades sino también en sus familias de origen y en las suyas propias, sus ingresos, la variación en cuanto a la edad, a la posición en el hogar, la salud, y quizás el número de hijos, el hecho de que tienen una relación variada con los hombres y chico de acuerdo con las estructuras de género y propias masculinas, los ciclos de la vida... (Rogers, 1980). El estatus de las mujeres, tal y como era concebido desde estas medidas, encubría más que aclaraba la posición de las mujeres de Tanganyika, incluyéndolas a todas en una única categoría y situación social, ocultando procesos más complejos de la propia estratificación social y de género de la estructura social en Lushoto (Fleuret, 1979).

En las Usambaras, que son parte de la cadena montañosa The Eastern Arc formada hace unos 100 millones de años (Woodcock, 1995), se extienden por toda la parte noreste del

1. El concepto de empoderamiento ha sido ampliamente discutido por distintos autores, desde los que se pone especial énfasis en las diversas formas en que las agencias del desarrollo han reconceptualizado el término con un contenido propio y distinto al original, siendo este último producto de los movimientos de mujeres de base del Sur de Asia. Sin embargo, no se profundizará en estos debates al no ser el objeto principal de discusión de este documento y por obvios motivos de espacio. Para una mayor profundización sobre el término ver Batliwala, 1994; Kabeer, 2001; Cronwall, 2005. 
país y, donde se sitúan entre otros distritos el de nuestro territorio etnográfico, el distrito de Lushoto, existían dos categorías sociales en el periodo precolonial: los campesinos y los esclavos (prisioneros de guerra o hijos de brujas). Por otra parte, los jefes tradicionales, eran los más ricos, poseyendo grandes cantidades de ganado $\mathrm{y}$, como otros hombres ricos en el territorio distribuían su riqueza, ganado, para reducir los riesgos de enfermedades o de ataques que le quitaran todo el ganado, previendo también provocar envidias. Los jefes en algunas ocasiones podían pedir que se pagara tributo a aquellos hombres que tuvieran gran cantidad de ganado. Además, cómo propietario de la riqueza colectiva de la tierra, proveía refugio y garantizaba el acceso a huertas para cultivo a los individuos y grupos sociales locales que se encontraban en márgenes extremos de supervivencia. En este sentido, como explica Ferran Iniesta los reyes clánicos y jefes tradicionales africanos solían tener más obligaciones que privilegios, donde "la jerarquía se inscribe en las exigencias del grupo para su supervivencia" (Iniesta, 2010: 59). La organización social se regía por descendencia patrilineal y residencia patrilocal. El matrimonio y la familia de origen eran entendidos como lugares de protección y seguridad para las mujeres y, si una mujer decidía no seguir viviendo con su marido, y si no encontraba refugio en su familia de origen, con su padre o hermano, podía refugiarse en la corte del jefe. Los hombres y las mujeres tenían derechos diferentes sobre la comida producida, y a pesar de que ambos géneros eran reclamados para trabajar en las huertas de los jefes tradicionales, aquellas mujeres maduras quedaban exentas al hacerse cargo de las huertas familiares y de los hogares. Los hombres trabajan en la preparación inicial de la huerta, las mujeres eran responsables de la cosecha. Las mujeres usaban la cosecha de sus huertas para alimentar a sus hijos y vendía el excedente. Los maridos cultivaban alimentos para el suministro en el hogar si el de sus esposas era insuficiente. El hogar era la unidad comprendida por una esposa y sus hijos perteneciendo a unidades familiares extensas, siendo comunes las familias poligámicas. Si el esposo moría, a cada hogar le correspondía ganado, y una mayor cantidad de éste se dirigía al hijo mayor de la esposa más antigua. La edad era uno de los principios diferenciadores más importantes entre las mujeres, situando a las de mayor edad en situaciones privilegiadas frente a las más jóvenes (Feierman, 1990).

Sin querer idealizar estas formas de organización social podemos afirmar que los planes de desarrollo social las alteraron y contemplaron la formación de las mujeres en términos occidentales, es decir como medios para colocarlas en lugares entendidos como los naturales del género femenino,

"En la formación de las mujeres, con el amable permiso del Departamento de Educación, se han realizado arreglos para usar la iglesia como la Escuela de Recursos Naturales de Tengeru, y empezar la primera fase de la formación de mujeres en el territorio, como parte del programa de formación que UNICEF comenzó. Veinte mujeres y chicas, todas ellas serán en el futuro empleadas por las autoridades locales [...] Al mismo tiempo las Oficiales 
del Desarrollo Social de las Mujeres continúan llevando a cabo los cursos de formación, para las Autoridades Nativas y para el personal temporal de los trabajos de instrucción con los clubes de mujeres y los clubes para líderes".

La visión de las mujeres nativas como criaturas oprimidas, a las que había que liberar a través de la civilización y el progreso, para convertirlas en señoras era el fundamento de las acciones que estos clubes llevarían a cabo, y que fueron integrados en la mentalidad de las mujeres y uno de los aspectos destacados entre las participantes,

"Ibamos allí para aprender a coser, a limpiar... eso te ayudaba a que tu marido estuviera más contento. Antes no sabíamos hacer nada. Yo me divorcié, él bebía mucho y no aguanté más" (Mujer 1. Participante en campañas de alfabetización y en los clubes de mujeres).

Las ideologías que transmitían eran aquellas subyacentes en los sistemas de subordinación de género europeas, donde las mujeres estaban destinadas al hogar y a la realización de unas tareas para el disfrute y felicidad de sus maridos, lo que nos lleva al segundo problema sobre el estatus de las propias mujeres europeas, espejo en el que pretendían que las mujeres africanas se mirasen. En este sentido, en partes de África, las mujeres y los hombres, no mantienen estatus unitarios, el género es sólo una de sus características definitorias. Mujeres y hombres pueden relacionarse jerárquicamente en uno o más de sus estatus recíprocos, pero no en otros (Sudarkasa, 1986). En cambio, en Gran Bretaña, tras la Segunda Guerra Mundial y segunda mitad del siglo XX, se produce una vuelta al énfasis en la familia nuclear como el modelo familiar a seguir. En este modelo, las tareas del hogar representan la ocupación principal de las mujeres, a pesar del activo role que tuvieron como trabajadoras fuera del hogar durante la Gran Guerra (Pugh, 1990:158). El matrimonio era el destino que toda mujer debía perseguir, predominando la vuelta a una visión conservadora del papel de las mujeres en la sociedad (Bruely, 1990). De acuerdo con autoras como Leonore Davidoff que sostienen que la privatización que implicó el capitalismo industrial fue parte del cambio en la visión sobre la posición de hombres y mujeres en el cosmos y en su relación con la naturaleza. La visión de la sociedad Victoriana sobre el mundo que ha predominado ha sido la de la creación de personas en distintas posiciones de poder y, que tienen los recursos y la necesidad para transmitir su posición como un aspecto central en su existencia. Desde esta percepción, las categorías de personas que están más alejados de los centros de toma de decisiones se clasifican en consecuencia, y también son visualizados en imágenes que hacen hincapié en su impotencia y degradación, así como con efectos contaminantes sobre las personas más cerca del centro de los que se aprovechan de su trabajo. La clase media victoriana, asoció la falta de poder de los grupos periféricos con un origen fisiológico. Esta asociación se utilizó como ideología dominante para mantener a estas personas en posiciones de menor consideración social (Davidoff, 1979). En este sentido, como parte de la tradición teológica cristiana la sociedad victoriana justificaba la posición subordinada de las 
mujeres asociándolas a su "carnalidad" potencial. La feminidad era asociada al cuerpo, por lo que las mujeres debían estar subordinadas a los hombre "como un trozo de carne debía estar al espíritu según el adecuado orden de la naturaleza" (Ruether, 1974). Las mujeres victorianas no sólo se las dividía entre las categorías de mujeres trabajadoras y clase media sino que también eran divididas entre señoras (ladies) y mujeres (women), categorías que no solo implicaban entender el género con un significado económico sino también social. Esta visión dual se mezclará con otras polaridades como las de blancos y negros, familiar y extranjero, el hogar y el imperio... (Davidoff, 1979). En la literatura de la época se exponían tales polaridades donde los nativos de las colonias eran representados cómo la naturaleza, el caos, la fecundidad, la diversión y, con lo negro, con la oscuridad. Las mujeres negras eran percibidas como exógamas, salvaje, donde su mejor concepción era la de una mujer natural, sensual, digna, y la peor como aquella con falta de conciencia y pérdida de significado (McNelly, 1975).

Los clubes marcaban lo que las mujeres, en general, debían o no debían ser y hacer sin tener en cuenta la posición que ya ocupaban en sus sociedades, y de manera diferenciada entre unas y otras. La imposición de este modelo en términos de tratamiento diferencial de hombres y mujeres y entre las propias mujeres debe considerarse como un proceso de discriminación (Rogers, 1980:35) dado que esta percepción colonial de las mujeres anulaba los conceptos de autonomía e independencia de las acciones de las mujeres tanzanas en periodos anteriores, como en el caso de las tareas agrícolas y comerciales. Las ideas que con estas medidas se trató de imponer, erosionaban los activos roles que las mujeres tenían como agricultoras y comerciantes. Una de las mujeres entrevistadas relató cómo trabajaba cuando era niña, de manera activa junto con sus padres, en las actividades agrícolas y comerciales,

"Yo trabajaba con mi padre, me iba con él a vender habichuelas al mercado cuando cosechábamos, además de trabajar en la huerta, claro... En esa época iba toda la familia, había que recoger los frutos del campo. Las mujeres y la azada siempre hemos sido amigos" (mujer entrevistada 5).

Sin embargo, estas ideas sobre el lugar de las mujeres junto con la introducción de cultivos comerciales, modificó negativamente la división sexual del trabajo que dio acceso a los beneficios de estos cultivos a los hombres discriminando a las mujeres. La producción de cultivos comerciales se conformó como dominio masculino. La exclusión de las mujeres de la producción comercial las privó también de las enseñanzas sobre modernas técnicas agrícolas que elevaban la producción. Con el desarrollo de cultivos comerciales y la expansión del mercado de materias primas, el trabajo de las mujeres, que permanecieron en la agricultura de subsistencia para el suministro en el hogar, se transformo en inferior y privado: no producía dinero para su introducción en la economía comercial y su función quedaba ahora limitada al grupo doméstico (Boserup, 
1970; Bryceson, 1993; Mbilinyi, 1980). La continua expansión de la economía de mercado ha favorecido la producción comercial, y con la demarcación de esta división sexual del trabajo las mujeres han tenido mayores dificultades que sus congéneres masculinos para poder apropiarse de los beneficios de esta producción, como resultado las hizo más dependientes económicamente de los maridos, situándolas en lugares de mayor vulnerabilidad (Berger y White, 1999),

"Nuestros hombres se iban a cultivar café y nosotras nos quedábamos cuidando de la casa $y$ de las huertas. Algunas iban a plantaciones cercanas pero la mayoría nos quedábamos aquí en la casa, porque no se nos permita salir, ¿quién iba a cuidar entonces de las huertas, de los animales...?" (Mujer entrevistada 7).

\section{LAS IDEOLOGÍAS PATRIARCALES COLONIALES Y LOS SISTEMAS DE ESTRATIFICACIÓN SOCIAL FEMENINA}

Las ideologías de género europeas impuestas marcaron la división entre la esfera pública y privada (Rodgers, 1980). La primera era definida como la esfera de lo económico, de las relaciones mercantiles y productivas, y la segunda como lo doméstico, lo invisible, lo improductivo. Las ideologías patriarcales existentes, entendidas como aquellas formas relacionales de control y dominación de las capacidades, privilegios y poderes femeninos (Nzegwu, 2000) reforzaron, en general, estas ideologías a su conveniencia. El modelo de familia que se desprendía como construcción y representación ideológica reforzaba un modelo familiar nuclear y heterosexual donde el hombre es el cabeza de familia, único proveedor y administrador de los recursos en el hogar (Rodgers, 1980; Kabeer, 1998),

"Me enviaron a Baga para casarme con un hombre que no conocía. Entonces en la mitad del camino escapé. Anduve durante toda la noche por el bosque. Al llegar el día vi la iglesia de San Martí, y fui para refugiarme, pero el párroco mandó avisar a mis padres que volvieron a enviarme a Baga. Al día siguiente me casaron... Después de unos años empezó a beber y a beber, y me pegaba. Cuando mis hijos se hicieron mayores me ayudaron a salir de aquella casa y me trajeron aquí de nuevo" (Mujer entrevistada 2).

"Cuando me casé, mi marido no quería que participara en ninguna actividad política, quería que estuviera en casa con todo preparado para él cuando volvía de hacer sus cosas... Pero aún así yo participé...Antes te casabas con quien elegía tu padre, tu no decidías nada, no es cómo ahora que se encuentran en las escuelas. Antes el gusto era el de tus padres, ellos decidían por ti" (Mujer entrevistada 5).

La idea de que los roles de género occidentales eran naturales se usó para apoyar las ideologías africanas masculinas que buscaban excluir a las mujeres de muchas de las nuevas áreas de la vida moderna introducidas por el mandato colonial (Davidoff, 1979; Otner, 1979; Rodgers, 1980). A pesar de que el mandato colonial no destruyó las unidades sociales en Tanganyika funcionó para reproducirlas y transformarlas. Parece que unas de 
las unidades sociales de mayor preocupación para los colonizadores en el control de las mujeres fueron los sistemas de parentesco. Los colonizadores construyeron las existentes relaciones de parentesco y buscaron su transformación cuando estas relaciones no aseguraban que las mujeres encajaban en los roles de género que los europeos sentían como apropiados. Así los hombres ancianos y los colonizadores británicos, pasaron las leyes costumarias que intentaban mantener a las mujeres bajo el control de sus linajes (Berger y White, 1999). Las comunicaciones entre misioneros del Distrito de Lushoto, el Comisionado del Distrito y el Comisionado del vecino Distrito de Moshi así lo relatan:

"Querido Sr. Piggott,

Tengo su carta respecto a Marta, hija de Yoswa, asistente de matrona en Mlalo, con respecto a la cual he hablado con su pastor la Reverenda Olimpa Shauri. Su marido Martini fue ordenado por la Corte Nativa pagarle sesenta shillings por la falta en su apoyo. Él esta preparado para hacerlo pero una cosa penosa es que ella por su parte no quiere aceptar ese dinero no deseando tener a este hombre nunca más. La iglesia por supuesto no puede tolerar esta actitud por su parte. Debo instar que todo lo necesario sea hecho para su reconciliación" (Carta de la Iglesia de Luandai al Comisionado del Distrito de Lushoto, 10 de Abril 1948).

"Un mchagga que trabajaba para Grewal Saw Mills, Ltd. Ha seducido a la esposa de otro hombre, una msambaa llamada Harriet d/o Salu y ha vuelto al Distrito de Moshi. Su dirección es Mamba, Marangu en Lushoto.

Le agradecería si pudiera ayudarle en el esfuerzo para recuperar a su esposa" (Carta del Comisionado del distrito Lushoto al Comisionado del Distrito de Moshi, 31 de Enero de 1953).

Estas medidas no afectaron de la misma manera a todas las mujeres del territorio, pero sí profundizaron la diferenciación social entre éstas. Si bien, existían distintas formas de estratificación antes de la colonización, con la intrusión europea se aceleró la división de la población en clases sociales, y también la diferenciación de clase entre las mujeres en Lushoto, remarcando las tensiones prevalentes entre las zonas urbanas y rurales (Hafkin y Bay, 1976; Bryceson, 1993). Las campañas de alfabetización y los clubes femeninos afectaron principalmente a las hijas y esposas de líderes y funcionarios africanos que conformaban parte del servicio colonial, residentes en las zonas urbanas cercanas a las residencias de los colonos. La diferenciación social entre las mujeres comenzó a hacerse más patente con la introducción de estas medidas coloniales que utilizaron los recursos de las misiones cristianas en la consecución de sus objetivos.

"Si, yo participé en esos talleres. Mi familia era musulmana, pero mi padre que era el jefe de la aldea, los británicos le dijeron que tenía que convertirse al cristianismo y que nosotras teníamos que participar en estas actividades. Y así lo hicimos... Gracias a eso me hice 
profesora, estudié, porque mira las mujeres de donde tú vives, las de Shashui, musulmanas, ellas no tienen nada, no tienen cultura, solo la agricultura y dependen de si llueve o no, y de sus maridos para vivir. Una mujer independiente tiene que vivir por sí misma, tener educación, su casa..." (Mujer entrevistada 1. Participante en los clubes).

"Los Wasambaa no quieren que las niñas estudien, pero mi padre era el Jefe de Mbuji. El Gobierno le dijo que tenían que enviar a los niños y niñas a estudiar y que los jefes tenían que enviar a sus hijos para dar ejemplo. Estudié en Mbuji, en el colegio primario y secundario, en las escuelas que construyeron los misioneros luteranos. Mi familia era musulmana pero para poder estudiar en estas escuelas había que convertirse al cristianismo. Los misioneros les decían que solo los cristianos podían estudiar luego. Cuando terminé me enviaron a la escuela de profesores de Dodoma" (Mujer entrevistada 2).

El gobierno británico colonial subvencionó las escuelas cristianas para la educación africana frente a las musulmanas, privilegiando a las primeras. En las Usambaras, los musulmanes, con excepción de los hijos de los líderes fueron excluidos de niveles superiores educativos (Ferierman, 1990). Algunas autoras han evidenciado cómo estas medidas fueron utilizadas, en ocasiones, por parte de las mujeres para subvertir el poder patriarcal de sus sociedades (Adams, 2006; Tripp, 2004). En Tanganyika sirvió para profundizar la diferenciación de clases entre aquellas mujeres africanas urbanas, cristianas, "occidentalizadas", y las mujeres africanas de las zonas rurales de comunidades islámicas. La participación en estas medidas benefició a las primeras situándolas en posiciones privilegiadas dentro de la escala social y ocupacional de las nuevas clases emergentes en el periodo post-colonial. A principios de la década de 1950 en Tanganyika había una clara distinción entre el estilo de vida de las mujeres mayoritariamente musulmanas analfabetas y aquella minoría de mujeres cristianas quienes habían aprendido algo de inglés en las escuelas cristianas. Estas disposiciones crearon diferencias que ilustran, a través de los diferentes hechos, cómo las ideologías de género quedarán vinculadas a la emergencia de las asunciones de clase y a las diferentes aspiraciones de estas mujeres (Geiger 1997:40),

"Me enviaron a estudiar educación y luego durante ujamaa fui la profesora de primaria en Shashui" (Mujer entrevistada 1).

\section{LAS DIFERENTES POSICIONES DE LAS MUJERES EN EL MOVIMIENTO DE RESISTENCIA COLONIAL}

Las precondiciones indispensable para la aparición de los nacionalismos en África fueron: los cambios económicos (cambio de una economía de subsistencia a una monetaria, crecimiento de la fuerza de trabajo asalariada, aumento de una nueva clase media), sociológicos (urbanización, movilidad social, educación occidental), religiosos e ideológicos (evangelización cristiana, abandono o frustración por los elementos 
educados occidentales), políticos (eclipse de las autoridades tradicionales, forjamiento de nuevos símbolos nacionales) que el colonialismo europeo impuso en el continente (Coleman, 1954:410-412). Todo esto fraguó de manera especial en Tanganyika donde las resistencias contra las políticas y medidas coloniales comienzan a partir de 1955 debido a varias razones, a saber: por una parte, por la arbitrariedad y abuso de los líderes y jefes tradicionales en la ejecución de las medidas de desarrollo agrícola; la desigual recaudación de impuestos que beneficiaba a algunos estratos sociales y perjudicaba a otros; por otra parte, la desigualdad creada por los distintos niveles de participación en las medidas sociales como educación y el diferente disfrute de las nuevas infraestructuras y servicios de las que las poblaciones urbanas gozaban frente a las agricultores campesinos que veían empeorar sus condiciones de vida. Estos últimos habían sufrido la exclusión y alienación de sus tierras y habían sido forzados a llevar a cabo agresivas medidas agrícolas. En la vida de las mujeres rurales, aumentó el volumen de su trabajo y la competitividad por la tierra dejándoles los peores suelos para el cultivo; se las marginó en la producción comercial y se las excluyó de las medidas educativas frente a los hombres y una minoría de mujeres cristianas (Tripp, 2004). En 1938 sólo un 4.34 por ciento de las 520,000 chicas en edad de escolarización iban a las escuelas, la mayoría de ellas en escuelas de las misiones. Las chicas musulmanas constituyeron sólo el 3 por ciento en las escuelas gubernamentales (Trebon, 1980). A finales de 1946, no había ninguna mujer entre los 917 estudiantes de la escuela secundaria en Tanganyika. En 1956, 204 chicas y 2409 chicos asistían a las escuelas de secundaria. En el este del país, las escuela gubernamentales supusieron sólo un tercio, mientras que el resto eran controladas por las escuelas católicas y protestantes (Mbilinyi, 1980). Las mujeres en general en Tanganyika estuvieron al menos dos generaciones detrás de los hombres en el acceso a la educación (Mbilinyi, 1980; Geiger, 1997; Tripp, 2004), particularmente las mujeres musulmanas tuvieron mayores dificultades para el acceso a las escuelas dada la prominencia de las escuelas cristianas en el territorio y, a pesar de que algunos padres apoyaban la educación de sus hijas:

"Empecé a ir a la escuela, era de los misioneros, me quedaba en casa de una pariente de mi padre. Pero un día cuando fui al colegio estaban cocinando cerdo y yo salí corriendo. Mi padre, cuando se enteró, no me dejó volver nunca más" (Mujer entrevistada 5, Lushoto).

La diferente participación en estas medidas profundizó los sistemas de diferenciación social entre las mujeres. Aquellas mujeres cristianas que accedieron a las escuelas y/o clubes de mujeres absorbieron e interiorizaron los discursos de la modernidad que las situaba en los espacios del hogar como madres y esposas, y limitaba su participación política. Por el contrario, en Lushoto, las musulmanas, que no tuvieron acceso a estas medidas coloniales se revelaron frente al poder colonial apoyando las iniciativas de independencia (Geiger, 1997). 
"Los británicos nos ayudaron, sí, al menos trajeron educación, y además nos enseñaron cómo comportarnos, antes íbamos casi con taparrabos. Trajeron desarrollo" (Mujer entrevistada 3)

"Ellos [se refiere a los colonos] pensaban que éramos animales" (Mujer entrevistada 4, musulmana, sin educación)

De esta manera, si los hombres educados por las iniciativas coloniales en las escuelas cristianas, ocuparon visiblemente el liderazgo de los movimientos de independencia, fueron las mujeres rurales, musulmanas sin educación, precisamente las más desfavorecidas por las políticas coloniales y sus medidas de desarrollo social, las que apoyaron tales movimientos. En ellas, el sentimiento nacionalista expresado como desafección hacia las autoridades y políticas del momento (Wrigth, 1993; Maddox y Giblin, 2005), creció ostensiblemente.

Hoy en día las diferencias sociales entre cristianos y musulmanes persisten en Lushoto. Así lo relataban dos de las mujeres entrevistadas:

"Entre los musulmanes las mujeres no poseen nada, no heredan y tiene que discutir sobre el tipo de cultivo que quieren cultivar con su marido y también pedir permiso para utilizar la tierra. Dice que esto deja en mala situación a las mujeres porque si se casan y se van pero luego vuelven no tiene nada para poder comer. Que si el marido muere tiene que pedir permiso a la familia del marido para quedarse en la casa y usar la tierra" (Mujer entrevistada 3).

"Los musulmanes no tenemos nada. Los cristianos reciben ayudas si no es del gobierno, es de la iglesia, pero nosotras nada" (Mujer entrevistada 4).

A partir de la década de 1950, las oposiciones al régimen colonial fueron fuertemente reprimidas. Un ejemplo de ello, fue la Ordenanza de Registro de Sociedades de 1954 donde se denegaba el registro a cualquier colectivo con actividades políticas (Feierman, 1990; Iliffe, 1979). Las mujeres que, según la ideología colonial, quedaban fuera de tales actividades por su roles femeninos, la usaron para saltarse estas restricciones y quedarse al margen de toda sospecha, haciendo de intermediarias entre las voces críticas del régimen colonial y las organizaciones políticas que buscaban la independencia del país, sobre todo la organización Tanganyika African Association (en adelante TANU). Sus acciones y relaciones dieron forma al movimiento nacionalista tanzano y a la movilización de las acciones de TANU de la época,

"En aquella época esperábamos a que llegara la noche. Yo salía de casa y esperaba en un árbol a que llegara mi amiga. Las dos íbamos a Mlalo a las reuniones clandestinas, recogíamos papeletas del partido que traíamos de vuelta aquí y las repartíamos entre nuestros vecinos. Así es como la gente se enteró de lo que estaba pasando y cómo ganamos" (Mujer 5, militante de TANU). 
"Las mujeres aquí fueron muy activas en los movimientos de aquella época, iban a los mítines clandestinos y distribuían panfletos del TANU, difundian las ideas del partido. Nadie se esperaba que ellas jugaran este papel, favorecieron mucho al movimiento. Las cosas en aquellos años estaban muy calientes" (Hombre entrevistado 1, militante de TANU).

A finales de los años 1950, las tensiones entre los oficiales del Gobierno británico y los nacionalistas africanos aumentaron. Durante las elecciones de 1958 treinta asientos representativos fueron ganados por miembros de TANU. La misión de Naciones Unidas de 1960 reconoció a TANU como organización política que mantenía el principio de igualdad democrática sin distinción de raza, sexo, lengua o religión. TANU solicitó un plebiscito para Tanganyika lo que llevaría finalmente al país a la Independencia en 1961.

Sin embargo, con respecto a las mujeres, TANU no incorporó a ninguna mujer a sus filas. Éstas fueron incluidas en el partido posteriormente, a petición de los antiguos colonizadores británicos. Bibi Titi Mohammed fue la primera mujer, musulmana, invitada a participar en la política como líder, e incorporó y unió a otras mujeres en el activismo político llegando a ser una figura central en este ámbito. Sin embargo, años después las diferencias de clase entre las mujeres emergieron en el partido, con la creación de una sección femenina en su interior, donde las mujeres educadas se hicieron cargo de los roles de liderazgo en estos departamentos de nueva creación. Mujeres comunes que desempeñaron un papel activo en la construcción de TANU quedaron fuera de las actividades políticas formales en el periodo postcolonial de Tanzania (Geiger, 1997). Con la declaración socialista, ujamaa, en 1967, y los primeros años de partido único se hicieron esfuerzos por disipar las distinciones entre musulmanes y cristianos, creando una nación sin divisiones raciales y religiosas (Glickman, 2011). La política socialista intentó crear la unidad nacional sin distinción tampoco entre hombres y mujeres estableciendo por ejemplo la universalidad de la educación primaria universal y gratuita ${ }^{2}$.

\section{CONCLUSIONES}

Las medidas de desarrollo social originaron y profundizaron los sistemas de desigualdad social entre las mujeres, y sus efectos sobre las identidades de género han sido divergentes. Por una parte, la ideología modernizadora trató de definir a una mujer africana al estilo europeo, domesticada en el interior del hogar con poco poder de decisión en la esfera pública. Sin embargo y contradictoriamente, aquellas que no accedieron a tales propuestas modernizadoras, principalmente las mujeres rurales musulmanas, encabezaron las

2. Hoy en día las discrepancias existen y las diferencias entre los géneros y confesiones también, sin embargo no podemos abordarlas por motivos de espacio. Sólo mencionaremos que algunos autores han llamado la atención sobre la apertura política con el pluripartidismo como una de las causas de surgimiento de las discrepancias y malestares en cristianos y musulmanes (Glickman, 2011), otros han discrepado por el hecho obvio de que el actual presidente del país, Jakaya Kikwete, es musulmán. Sin embargo, habría que realizar un análisis en profundidad para poder estar de acuerdo con cualquiera de estas posiciones. 
luchas por la liberación nacional, ocupando un papel primordial en la consecución de la independencia.

A pesar de los avances en la posición de las mujeres en Tanzania, como muestran algunos datos como por ejemplo el aumento de representación femenina en el parlamento que desde 1961 al año 2007 el número de mujeres ha pasado de ser del $8 \%$ al 20\% (EISA, 2007), el recorrido de éstas para lograrlo, ha estado marcado por la segregación de clase y religiosa. Las mujeres musulmanas parecen haber ocupado mayores cotas de activismo político a través de organizaciones de la sociedad civil que a través de la representación política parlamentaria (Dunbar, R. A., 2000).

Si bien los procesos de aculturación y diferenciación social no son lineales como hemos mostrado, en el caso de las medidas de desarrollo social implantadas en Tanganyika nos llevan a concluir, cómo el contacto con las ideologías de género coloniales generaron unas identidades específicas y diversas, que produjeron diferencias y desigualdades entre las poblaciones, en este caso entre las mujeres de Tanzania. Al mismo tiempo, estos procesos, fueron utilizados por las mujeres rurales islámicas manipulando estas ideas que las suprimían del campo político, participando en el movimiento que conduciría a la independencia del país. Por su parte, aquellas mujeres que accedieron a estas medidas se situaron en importantes posiciones dentro de las nuevas clases urbanas emergentes del nuevo país tras la independencia. 


\section{REFERENCIAS BIBLIOGRÁFICAS}

Adams, M. (2006) "Colonial Policies and Women's Participation in Public Life: The Case of British Southern Cameroons." African Studies Quarterly 8 (3) http://web.africa.ufl. edu/asq/v3/v8i3a1.htm [Consultado el 17 de diciembre de 2010].

Adejumboi, S. (2000) Between Democracy and Development in Africa: What are the Missing Links? University of Cape Town: Centre for African Studies.

Adekson, J.B. (1981) “The Algerian and Mau Mau Revolts: a Comparative Study in Revolutionary Warfare”. Comparative Strategy, 2 (1), pp. 69-92.

Aina, T. A. (1999) "West and Central Africa: social policy for reconstruction and development". En Morales-Gomez (Ed.) Transnational Social Policies: The New Development Challenges of Gobalization. Ottawa: International Development Research Centre.

Batliwala, S. (1994) “The Meaning of Women's Empowerment: New Concepts from Action". En G. Sen, A. Germain and L. C. Chen (eds.) Population Policies Reconsidered: Health, Empowerment and Rights. Boston: Harvard University Press, pp. 127-138.

Berger, I. y White, F. E. (1999) Women in sub-Saharan Africa: restoring women to history. Indiana: Indiana University Press.

Boserup, E. (1970) Women's Role in Economic Development. Nueva York: St. Martin’s Press.

Bradley, K., y Khor, D. (1993) "Toward an integration of theory and research on the status of women". Gender and Society, 7 (3), pp. 347-378.

Bruley, S. (1999) Women in Britain since 1900. Houndmill: Macmilan.

Bryceson, D.F. y Mbilinyi, M. (1978) The changing rol of Tanzanian Women in production: from peasants to proletarians. Dar es Salaam: University of Dar es Salaam Press.

Bryceson, D.F. (1993) Liberalizing Tanzania’s Food Trade: Public \& Private Faces of Urban Marketing Policy, 1939-1988. London: James Currey Publishers.

Carrasco, C. y Mayordomo, M. (2000) "Hacia una nueva metodología para el estudio del tiempo y del trabajo". Comunicación presentada en Taller Internacional Cuentas Nacionales de Salud y Género, Santiago de Chile 19 de Octubre 2001, http://www.paho. org/Spanish/HDP/hdw/chile-cric.PDF [Consultado el 18 de diciembre de 2010]. 
Coleman, J. (1954) "Nationalism in Colonial Africa." American Political Science Review XLVIII, pp. 404-26.

Comas D'Argemir, D. (1998) Antropología Económica. Barcelona: Arial.

Cornwall, A. y Brock, K. (1995) Beyond Buzzwords "Poverty Reduction", "Participation" and "Empowerment" in Development Policy. Paper no. 10, Geneva: UNRISD.

Creighton, C. y Omari C. K. (Eds) (1995) Gender, family and household in Tanzania. Aldershot: Avebury.

Davidoff, L (1979) "Class and Gender in Victorian England: The Diaries of Arthur J. Munby and Hannah Cullwick". Feminist Studies, Vol. 5, No. 1, Women and Power: Dimensions of Women's HistoricalExperience (Spring, 1979), pp. 86-141.

Dunbar, R. A. (2000) "Muslim women in African history. En N. Levtzion y R. L. Pouwels (Eds.) The History of Islam in Africa. Athens: Ohio University Press.

Ekejiuba, F. (1995) "Down to fundamental: Women-Centred Heartholds in Rural West África”. En D. Bryceson. (ed.) Women Wielding the Hoe: Lessons from Rural África for Feminist Theory and Development Practice. Oxford: Berg Publishers.

Feierman, S. (1990) Peasant Intellectuals. Anthropology and History in Tanzania. Wisconsin: The University of Wisconsin Press.

Fleuret, A. (1979) "The role of wild foliage plant in diet: A case study from Lushoto, Tanzania”. Food Nutrition 8, pp. 87-93.

Geiger, S. (1997) TANU Women Gender and Culture in the Making of Tanganyikan Nationalism, 1955-1965. Portsmouth: Heinemann.

Glickman, H. (2011) "The Threat of Islamism in Sub-Saharan Africa: The Case of Tanzania”. Foreign Policy Research Institute http://www.fpri.org/enotes/201104. glickman.islamismsubsaharanafrica.pdf [Consultado el 11 de Julio de 2012].

Hafkin, N. y Bay, E. (1976) Women in Africa. Stanford: Stanford Univ. Press.

Iliffe, J. (1967) "The Organization of the Maji Maji Rebellion". Journal of African History, 8 (3), pp. 495-512.

Iliffe, J. (1979) A Modern History of Tanganyika. Cambridge: Cambridge University Press. 
Iniesta, F. (2010) El pensamiento tradicional africano. Barcelona: La catarata.

Kabeer, N. (1998) Realidades trastocadas las jerarquías de género en el pensamiento del desarrollo. Mexico: Paidos.

Kabeer, N. (2001) "Reflections on the Measurement of Women's Empowerment". En Discussing Women's Empowerment: Theory and Practice. Swedish SIDA.

Linton, R. (1936) The Study of Man. New York: Appleton-Century.

MCnelly, C. (1975) "Nature, Women and Claude Levi-Strauss: A Reading of Tristes Tropiques as Myth”. Massachusetts Review 16 (Winter 1975), pp. 10-11.

Maddox, G. H. y Giblin, J. L. (2005) In Search of a Nation. Histories of Authority and Dissidence in Tanzania. Oxford: Eastern African Studies.

Mbilinyi, M. (1980) African education in the British Colonial Period. En Tanzania under colonial rule. Londres: M. Kaniki.

Mbilinyi, M.; Rusimi, M.; Chachage, S.L. (2003) Activist Voices. Feminist Struggles for an Alternative World. Dar es Salaam: TGNP.

Moore, H. (1991) Antropología y feminismo. Madrid: Cátedra.

Narotzky, S. (2004) Antropología Económica. Nuevas Tendencias. Barcelona: Melusina.

Nelson, J. (1996) “The Masculine Mindset of Economic Analysis", http://www.facstaff. bucknell.edu/jshackel/iaffe/julienelson.html [Consultado el 17 de enero 2011].

Nzegwu, N. (2000) Family Matters: Feminist Concepts in African Philosophy of Culture. Albany: State University of New York.

Nzegwu, N. (2002) "Questions of agency: development, donors, and women of the south". Jenda: A Journal of Culture and African Women Studies, 2 (1) http://www.jendajournal. com/vol2.1/nzegwu.html [Consultado el 25 de enero de 2011].

Okongwu, A. F. y Mencher, J.P. (2000) "The Anthropology of Public Policy: Shifting Terrains". Annual Review of Anthropology 29, pp. 107-124.

Ortner, S B. (1979) “Es la mujer al hombre lo que la Naturaleza es a la Cultura?”. En Harris, O. y Young, K. (comps.) Antropología y Feminismo. Barcelona: Anagrama, pp.109-131. 
Oyewumi, O. (1997) The Invention of Women: Making an African Sense of Western Gender Discourses. Minnesota: University of Minnesota Press.

Pugh, M. (1990) "Domesticity and the decline of feminism 1930-1950". En Harlod L. Smiths A. (Eds) British Feminism in the Twentieth Century. Mass: University of Massachusetts Press.

Rogers, B. (1980) The Domestication of Women: Discrimination in Developing Societies. Great Bretain: Kogan Page Ltd.

Rosaldo, M. (1974) "Woman, culture and society: A theoretical overview". En Rosaldo and Lamphere (eds.). Woman. Culture, and Society. Stanford: Standford University Press.

Ruether, R. (1974) "Misogynism and Virginal Feminism in the Fathers of the Church". En Religion and Sexism: Images of Women in the Jewish and Christian Tradition. New York: Simon \& Schuster.

Spencer, S. (2005) Gender, Work and Education in Britain of the 1950s. Basingstoke: Macmillan.

Sudarkasa, N. (1986) "The Status of Women in Indigenous African Societies". Feminist Studies12 (1), pp. 91-103.

Trebon, J. (1980) Development of the pre-independence educational system in Tanganyika with special emphasis on the role of missionaries. Denver: Univerty of Denver.

Tripp. M.A (2004) "A New Look at Colonial Women: British Teachers and Activists in Uganda, 1898-1962”. Canadian Journal of African Studies / Revue Canadienne des Études Africaines, Vol.38, No. 1, pp. 123-156.

Vieitez, S. (2005) "Antropología y género: Miradas desde África”. Crítica 923:32-35 http://www.uv.es/iued/actividades/articulos/CriticaSOLEDAD.pdf [Consultado el 27 de diciembre de 2010].

Wedel, J. R. (2005) “Why an Anthropology of Public Policy?”. Anthropology Today, 21 (1), pp. 1-2.

Whyte M. K. (1978) The Status of Women in Preindustrial Societies. Princeton, N.J.: Princeton University Press.

Wright, M. (1993) Strategies of Slaves and Women: Life Stories from East/Central Africa. New York: L. Barber Press. 
Yanagisako, S.; Collier, J. (1994) “Gender and Kinship Reconsidered: Toward a Unified Analysis”. En Robert Borofsky (Ed.) Assessing Cultural Anthropology. Hawaii Pacific University: Mc Graw-Hill.

Zelditch, M, JR. (1968) “Social status". En David L. Sills (Ed.) International encyclopedia of social sciences. New York: Macmillan. 